\title{
Was bewegt die Führungskräfte? Von der Agency-Theorie zur Soziologie des Managements
}

\section{Von Martin Höpner}

\section{Problemstellung}

Warum orientieren sich Führungskräfte deutscher Großunternehmen seit den späten neunziger Jahren verstärkt an den Finanzinteressen der Aktionäre? Agency-Theoretiker argumentieren, dass die Interessen von Aktionären (den Prinzipalen) und Führungskräften (den Agenten) verschieden sind, und dass über das Vertragsverhältnis hinausgehende Instrumente vonnöten sind, um die mit der Unternehmensleitung beauftragten Manager auf die Verfolgung der Eigentümerinteressen zu verpflichten. Die von Agency-Theoretikern diskutierten Instrumente zur Disziplinierung von aus Investorensicht opportunistischen Führungskräften (Fama 1980; Jensen/Meckling 1976, S. 328-330; Jensen 1993, S. 850-854; Shleifer/Vishny 1996) klären einen erheblichen Teil der in empirischen Studien vorgefundenen Varianz auf. Deutsche Unternehmen verhalten sich um so aktionärsorientierter, ${ }^{1}$ je größer der Aktienanteil ist, der sich im Besitz von im Vergleich zu Privataktionären handlungsfähigeren institutionellen Anlegern befindet; je mehr die Eigentümerstrukturen der Unternehmen eine feindliche Übernahme ermöglichen; und je mehr der Absatz der Unternehmen der internationalen Konkurrenz ausgesetzt ist (Achleitner/Bassen 2000; Eckert 2004; Höpner 2003; Januszewski et al. 1999). Im Hinblick auf diese »Agency-Mechanismen« scheinen sich die aus den ökonomischen Modellen ergebenden Erwartungen zu bestätigen. Spezifisch soziologische Zugänge scheinen nicht notwenig, um das veränderte Verhalten der Führungskräfte zu verstehen.

Allerdings zeigt das Verhalten der Führungskräfte im Übergang zur aktionärsorientierten Unternehmensführung Merkmale, die aus den Vorhersagen der Agency-Theorie nicht abzuleiten sind und ihr - je nach Ausprägung - möglicherweise sogar widersprechen. Der ökonomischen Theorie zufolge haften Führungskräften Präferenzen zur Schmälerung der Aktienrendite durch unprofitables Unternehmenswachstum, Diversifizierung und Risikoaversion in Finanz- und Investitionsfragen sowie eine Neigung zur Selbstbereicherung an, die sie verfolgen, wenn ihr durch die Wirkung der Agency-Mechanismen definierter Handlungsspielraum dies zulässt. Demnach sollten Führungskräfte auf die mit Shareholder-Value-Politik einhergehende Einschränkung ihres Handlungsspielraums ablehnend reagieren. Tatsächlich aber ist das Gegenteil der Fall: Aktionärsorientierte Unternehmensführung genoss unter deutschen Führungskräften in den vergangenen Jahren ein hohes Ansehen und hatte damit den Charakter einer - im neutralen Wortsinn - Managementideologie, auf die externe AgencyMechanismen zwar befördernd gewirkt haben mochten, die in den meisten Fällen aber nicht gegen den Willen der Führungskräfte durchgesetzt werden musste.

1) Es wird hier davon ausgegangen, dass das Bekenntnis zu aktionärsorientierter Unternehmensführung seit den späten neunziger Jahren - und über den Einsturz der Börsenkurse im Jahr 2000 hinaus - materielle Veränderungen der Unternehmensführung nach sich gezogen hat. Zu Instrumenten aktionärsorientierter Unternehmensführung zählen beispielsweise die Nutzung wertorientierter, die Kapitalkosten berücksichtigender Steuerungsgrößen, die Implementation von segmentspezifischen Renditezielen, aktive Investor-Relations-Arbeit, Transparenz der Rechnungslegung, variable Entlohnung der Führungskräfte und der Rückkauf eigener Aktien in Phasen überschüssiger Liquidität. Im operativen Sinn zielt aktionärsorientierte Unternehmensführung vor allem auf die Vermeidung unrentabler Quersubventionierung und die Konzentration auf profitable Kerngeschäfte: »Investiere nur in Chancen mit einem glaubhaften Wertsteigerungspotential und zahle den Investoren dann Cash zurück, wenn wertsteigernde Investitionschancen nicht verfügbar sind« (Rappaport 1999: 112). 
Dass das Shareholder-Value-Konzept bei deutschen Führungskräften in den vergangenen Jahren in hohem Ansehen stand, lässt sich anhand unternehmensvergleichender Daten veranschaulichen. In Abbildung 1 wurde auf der Waagerechten das Ergebnis einer Imagestudie im Auftrag der Wirtschaftswoche abgetragen, in deren Rahmen 1.400 inländische Führungskräfte gebeten wurden, Großunternehmen hinsichtlich ihres Innovationsimages - im Sinne von neu, zukunftsträchtig und wachstumsfördernd - einzustufen (Wirtschaftswoche vom 1.6.2000, S. 96-107). Niedrige Werte bedeuten eine positive Bewertung des Images der Unternehmen, bei hohen Werten vergaben die Befragten schlechte Noten. Auf der Senkrechten wurde der Grad an Aktionärsorientierung in den späten Neunzigern abgetragen. Ein entsprechender Index ergibt sich durch Kombination von unternehmensbezogenen Daten zu den Investor Relations, der Bilanzierung, der Variabilität der Führungskräftevergütung und der Implementation von Renditezielen (Höpner 2003: 60-67). Beide Rangfolgen stimmen in beachtlichem Ausmaß überein ( $\mathrm{r}=-.66, \mathrm{p}=.000, \mathrm{n}=33)^{2}$ : Je ausgeprägter die Aktionärsorientierung der Unternehmen, umso besser war das Image, das ihnen von den befragten Führungskräften zugesprochen wurde.

\section{Schaubild 1: Unternehmensimage und Shareholder Value 33 Unternehmen, späte 1990er Jahre}

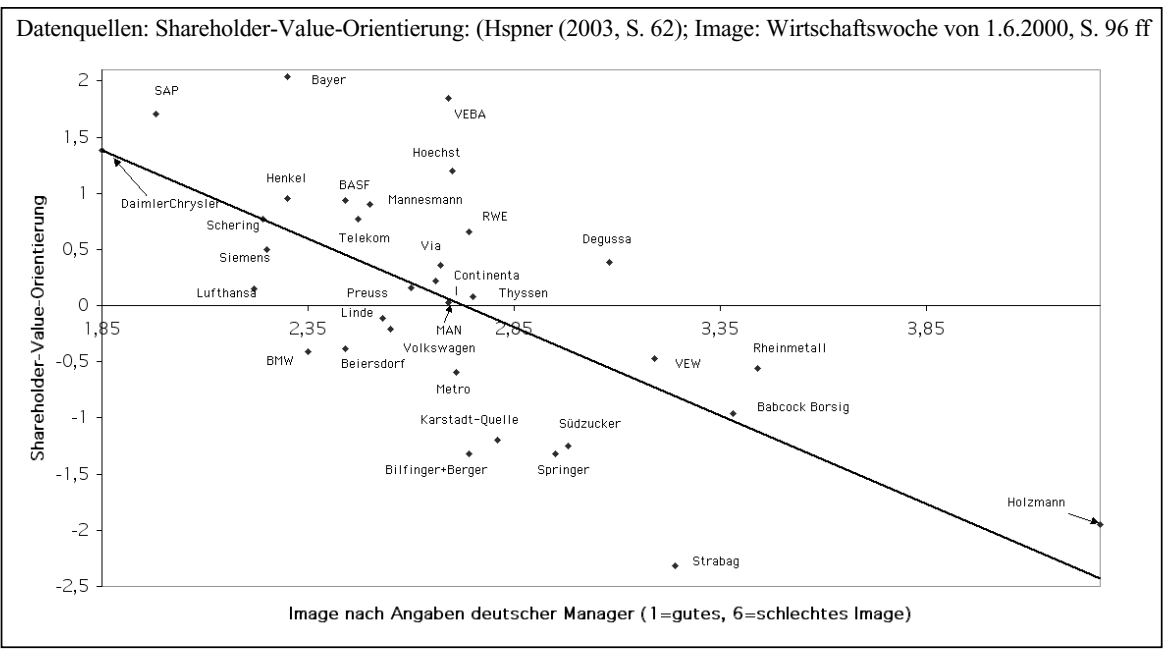

Es zeigt sich, dass die Frage nach den Motiven hinter der Orientierung der Manager an den Interessen der Aktionäre von der Agency-Theorie nicht wirklich beantwortet wurde: Warum sollten Führungskräfte Strategien zur Einschränkung ihres eigenen Handlungsspielraums, zur eigenständigen Anlegung von Fesseln gegen die Verfolgung ihrer (im Sinne der AgencyTheorie) »natürlichen« Präferenzen befürworten und ihnen ein positives Image zusprechen? Nachfolgend wird argumentiert, dass die Annahmen der Agency-Theorie mit Befunden aus der Soziologie des Managements verknüpft werden müssen, um zu einer befriedigenden Antwort auf diese Frage zu gelangen. Die Argumentation erfolgt in drei Schritten. Erstens wird gezeigt, dass während der neunziger Jahre Veränderungen in den Karrierehintergründen der Führungskräfte stattfanden, die die Übernahme von Strategien aktionärsorientierter Unternehmensführung begünstigten. Zweitens wird argumentiert, dass sich der unkontrollierte

2) Angegeben werden der Pearsonsche Korrelationskoeffizient, die Signifikanz und die Fallzahl. 
Handlungsspielraum der Führungskräfte in Wahrheit nicht vermindert, sondern qualitativ verändert hat: der zunehmenden Marktkontrolle steht rückläufige Insiderkontrolle gegenüber. Drittens wird dargelegt, dass der partielle Übergang von der externen zur internen Unternehmenskontrolle mit steigenden Managergehältern einhergeht und deshalb den Interessen der Führungskräfte entgegenkommt. Die Argumentation wird durch Datenanalysen gestützt, die sich auf die 40 größten Industrieaktiengesellschaften während der neunziger Jahre beziehen.

\section{Karrierewege im Wandel}

Nachfolgend werden Veränderungen in den Karrieremustern deutscher Topmanager aufgezeigt und deren Implikationen für die Unternehmenspolitik diskutiert. In der international vergleichenden Managementliteratur wurden Ausbildungs- und Rekrutierungsmuster deutscher Topmanager insbesondere den Karrieremustern von Führungskräften aus angloamerikanischen Ländern gegenübergestellt. Dabei zeigten sich für Deutschland folgende Charakteristika:

Im Unterschied zu angloamerikanischen Ländern existieren in Deutschland keine Elitehochschulen, die auf die Integration der Studenten in die Eliten der jeweiligen Länder ausgerichtet sind und wo Fertigkeiten wie Kommunikationsfähigkeit, Belastbarkeit und auch Elitebewusstsein trainiert werden (M. Hartmann 1997, S. 29; Lane 1989, S. 93). Eine spezielle Elitebildung findet in Deutschland kaum statt. Studienabschlüsse deutscher Manager dokumentieren Fachwissen statt Führungsfähigkeiten.

In der soziologischen Managementforschung wurde den typischen Ausbildungs- und Studiengängen von Topmanagern ein entscheidender Beitrag zur Konstitution der deutschen konsensorientierten und techniklastigen Produktionsweise zugesprochen. In Deutschland wurde ein vergleichsweise hoher Anteil an Managern festgestellt, die ein juristisches, technisches oder naturwissenschaftliches Studium oder eine Fachausbildung absolviert haben. Deutsche Manager gelten als Fachleute auf dem Gebiet der Produktion (Egan 1997, S. 6; Faust 2000, S. 67-68; H. Hartmann 1968; Lane 1989, S. 92; Sorge 1999; Steward et al. 1994, S. 21; Walgenbach/Kieser 1995, S. 278-279; Zapf 1965). Eine viel zitierte Untersuchung der Ausbildungswege von Vorstandsmitgliedern großer und mittlerer Unternehmen des verarbeitenden Gewerbes hat für die sechziger und siebziger Jahre ergeben, dass nur etwas über die Hälfte von ihnen ein Studium absolviert hatte. Davon waren knapp 60 Prozent Ingenieure und Naturwissenschaftler, 30 Prozent Wirtschaftswissenschaftler und 10 Prozent Juristen (Poensgen 1982, S. 17). Für angloamerikanische Führungskräfte ist hingegen eine Ausbildung im Rechnungswesen typisch.

Die Karrierewege amerikanischer Führungskräfte sind von starker Konkurrenz geprägt. Das zeigt deren im Vergleich zu Deutschland kürzere Verweildauer in ihren Ämtern. Die Bereitschaft, Führungskräfte bei unbefriedigender Performanz gegen einen Konkurrenten auszutauschen, ist in Deutschland geringer ausgeprägt.

Viele deutsche Vorstandsmitglieder haben eine Hauskarriere hinter sich, ihre Stellung also durch unternehmensinternen Aufstieg erreicht. Spiegelbildlich dazu erweist sich der unternehmensexterne Arbeitsmarkt für Führungskräfte weniger entwickelt als in englischsprachigen Ländern, wo es für Topmanager typisch ist, die Unternehmen mehrfach zu wechseln. Das führt zur Emanzipation von unternehmensspezifischen Bindungen (Bleicher 1983, S. 140).

Diese Besonderheiten in Ausbildung, Rekrutierung und Verweildauer verdichten sich zu einer spezifischen »sozialen Welt« von Topmanagern, die auch Grundentscheidungen über Weichenstellungen der Unternehmenspolitik beeinflussen. Solche Verknüpfungen zwischen Biographie- und Verhaltensvariablen sind in der soziologischen Managementliteratur häufig 
diskutiert worden. Dominante Karrieremuster prägen nicht nur die Fachkompetenzen von Führungskräften, sondern auch deren Identitäten und Wertorientierungen. Technische Ausbildungs- und Studiengänge verschaffen Facharbeitern und Führungskräften eine gemeinsame fachliche Basis, begünstigen die Kommunikation zwischen beiden Gruppen und wirken deshalb als integrierender Mechanismus (Sorge 1999, S. 25). Die Techniklastigkeit der deutschen Managementausbildung begünstigt zudem Managementphilosophien, bei denen der Einsatz moderner Technik und Wachstumserfolge anstelle von finanzieller Performanz und betriebswirtschaftlichem Denken im Vordergrund stehen (Bleicher 1983, S. 143; Byrkjeflot 2002; Sorge 1999). Es ist nahe liegend, dass auch die lange Verweildauer deutscher Führungskräfte in ihren Ämtern mit dem für Deutschland typischen langfristigen Horizont der Unternehmenspolitik in Verbindung gebracht wurde. Bei amerikanischen Managern können bereits Schwankungen in den Quartalszahlen Rückwirkungen auf die Arbeitsplatzsicherheit haben.

Gilt dieser Forschungsstand auch für die neunziger Jahre? Welche Entwicklungen haben sich in diesem Jahrzehnt vollzogen? Um dies herauszufinden, wurden die Biographien der 90 Führungskräfte erhoben, die in den neunziger Jahren Vorstandsvorsitzende der (nach Angaben der Monopolkommission) 40 größten börsennotierten Industrieunternehmen waren. ${ }^{3}$ Die Biographien wurden nach verschiedenen Fragestellungen in Variablen codiert. Mit Hilfe dieser Variablen konnten die Trends im Verlauf der neunziger Jahre nachgezeichnet werden. Insgesamt verteilen sich die (ausschließlich männlichen) 90 Topmanager auf 400 Beobachtungspunkte (40 Unternehmen mal 10 Jahre). Wegen fehlender Informationen reduzierten sich die validen Beobachtungspunkte auf 376 (24 fehlende Werte). Ausgewählte Ergebnisse werden in Schaubild 2 dargestellt.

\section{Schaubild 2: Karrierewege von Vorstandsvorsitzenden, ausgewählte Indikatoren. 40 Unternehmen, 1990-1999}

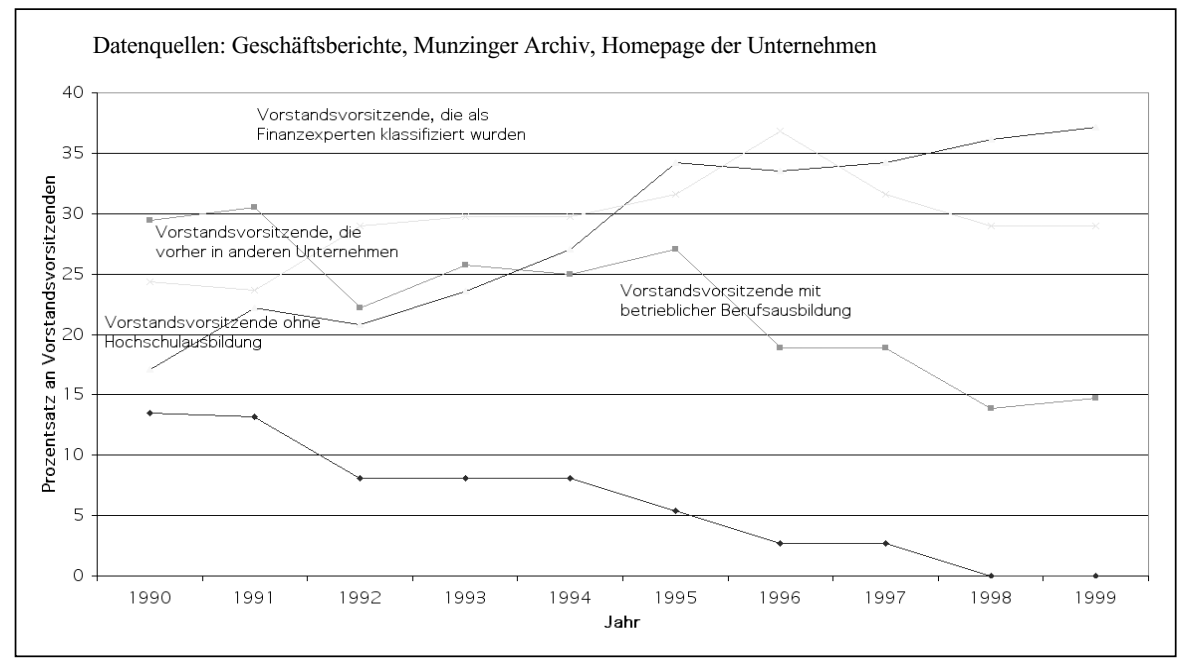

Es zeigt sich, dass es für deutsche Spitzenmanager entgegen der Erwartung nicht typisch war, eine betriebliche Berufsausbildung absolviert zu haben. Im Durchschnitt wurden die 40 betrachteten Unternehmen in den neunziger Jahren nur zu 23 Prozent (bezogen auf die vali-

3) Die Informationen stammen aus Geschäftsberichten, dem Munzinger Archiv, Zeitungsberichten und Homepages der Unternehmen. 
den Beobachtungspunkte) von Managern mit Ausbildungsberuf im Sinne des Berufsbildungsgesetzes $(\mathrm{BBiG})$ geleitet. In den sechziger und siebziger Jahren wurden noch weitaus höhere Anteile an Spitzenmanagern festgestellt, die eine Lehre absolviert hatten (M. Hartmann 1995, S. 454). Auffällig ist darüber hinaus, dass der Anteil an Vorstandsvorsitzenden mit betrieblicher Berufsausbildung in den neunziger Jahren weiterhin rückläufig war und sich von 30 Prozent im Jahr 1990 auf 15 Prozent im Jahr 1999 halbierte. Zudem befanden sich unter den Managern mit Berufsausbildung lediglich fünf Personen, die eine gewerbliche Ausbildung absolviert hatten. ${ }^{4}$ Der Rest entfällt auf kaufmännische Berufe. In den Karrieremustern deutscher Topmanager fand also ein deutlicher Bedeutungsverlust der beruflichen Ausbildung statt. Von einem bedeutenden Anteil Vorstandsvorsitzender, die ehemals gewerbliche Auszubildende waren, kann keine Rede sein.

Typisch für die Ausbildungswege der Vorstandsvorsitzenden war ein Studium, häufig mit anschließender Promotion. Insgesamt amtierten nur in sechs Prozent der erfassten Fälle Vorstandsvorsitzende ohne Hochschulstudium. Auch hier zeigt der Zeitverlauf einen eindeutigen Trend. Entfiel der Anteil Nichtstudierter zum Anfang der neunziger Jahre noch auf 14 Prozent, gab es im zu Grunde gelegten Sample in den Jahren 1998 und 1999 keinen Vorstandsvorsitzenden mehr, der kein Hochschulstudium abgeschlossen hatte. 59\% der Beobachtungspunkte entfallen auf promovierte Vorstandsvorsitzende. Das deutet auf einen Professionalisierungstrend im deutschen Topmanagement.

Anders als von der vergleichenden Managerliteratur für frühere Zeiträume festgestellt (z.B. Egan 1997, S. 6), ist bei den Studienrichtungen nun keine Dominanz technischer Fächer mehr zu beobachten. Wie sich zeigt, wurden die betrachteten 40 Unternehmen in den 10 beobachteten Jahren nicht einmal zu einem Drittel von Managern geleitet, die ein naturwissenschaftliches oder technisches Studium absolviert hatten (32 Prozent). Der häufigste Studiengang deutscher Führungskräfte waren in den Neunzigern die Wirtschaftswissenschaften (39 Prozent). 24 Prozent der Beobachtungspunkte entfallen auf Juristen. Im Verlauf der neunziger Jahre blieben die Anteile der jeweiligen Studienfächer relativ konstant. Alle Studienrichtungen gewannen leicht auf Kosten des Rückgangs der Nichtstudierten. Weil den naturwissenschaftlichen und technischen Fächern in früheren Studien noch eine wesentlich größere Bedeutung zugesprochen wurde (z.B. Poensgen 1982, S. 17), liegt die These nahe, dass bereits in den achtziger Jahren ein Bedeutungsverlust der naturwissenschaftlich-technischen Ausbildungsmuster stattfand. Auf die Gegenwart bezogen, ist das Bild des von Technikern beherrschten deutschen Großunternehmens offensichtlich ein Vorurteil.

Darüber hinaus erfolgte in den neunziger Jahren eine Stärkung finanzwissenschaftlicher Kompetenz. Für Schaubild 2 wurden Vorstandsvorsitzende als Finanzexperten klassifiziert, wenn ihr Karriereweg über das Finanzressort desselben oder eines anderen Unternehmens verlaufen war. Der Anteil dieser Finanzexperten, auf die insgesamt 29 Prozent der Beobachtungspunkte entfallen, stieg zwischen 1990 bis 1999 um etwa fünf Prozentpunkte. Es verwundert nicht, dass diese Vorstandsvorsitzenden in ihrer Mehrzahl studierte Wirtschaftswissenschaftler waren. Darüber hinaus fanden sich einige Juristen unter den Finanzexperten. Im betrachteten Sample gibt es kein Beispiel für einen Naturwissenschaftler oder Techniker, der über ein Finanzressort Vorstandsvorsitzender geworden wäre.

4) Joachim Milberg (BMW) hat eine Ausbildung zum Maschinenschlosser absolviert; Jürgen E. Schrempp (Daimler-Benz bzw. DaimlerChrysler) hat im selben Unternehmen, das er später leiten sollte, Kfz-Mechaniker gelernt; Heinz Ruhnau (Deutsche Lufthansa) ist gelernter Elektromaschinenbauer; Wolfgang Urban (Metro) hat eine Ausbildung zum Werkzeugmacher abgeschlossen; Fritz Ziegler (VEW) wurde im Bergbau ausgebildet. 
Ein massiver Wandel der Karrieremuster fand hinsichtlich der Unterscheidung zwischen internen und externen Rekrutierungen statt. In der vergleichenden Managementforschung wurde die geringe Bedeutung des externen Arbeitsmarkts und damit die Dominanz von unternehmensinternen Hauskarrieren aufgezeigt (Bleicher 1983, S. 141-143; M. Hartmann 1995, S. 464; Walgenbach/Kieser 1995, S. 279-280). Um dies zu überprüfen, wurden die Tätigkeiten der Vorstandsvorsitzenden in den fünf bis 15 Jahren vor ihrer Amtszeit betrachtet. Anschließend wurde zwischen internen und externen Herkünften unterschieden. ${ }^{5}$ Es zeigt sich, dass die Befunde der soziologischen Managementliteratur noch immer zutreffen. Der typische Karriereweg der betreffenden Vorstandsvorsitzenden führte durch das eigene Unternehmen (71 Prozent). In einigen Fällen erstreckte sich die Unternehmenszugehörigkeit über mehrere Jahrzehnte. ${ }^{6}$ Nur bei 29 Prozent der validen Beobachtungspunkte waren Vorstandsvorsitzende im Amt, die vorher in einem anderen Unternehmen tätig waren. Wie aus Schaubild 2 ersichtlich, verdoppelte sich der Anteil extern rekrutierter Manager zwischen 1990 und 1999 von 17 Prozent auf etwa 34 Prozent. Die Bedeutung des Arbeitsmarkts für Führungskräfte nahm also rapide zu.

Zusätzlich wird überprüft, ob sich Veränderungen bei den Verweildauern der Vorstandsvorsitzenden in ihren Ämtern beobachten lassen. Entsprechende, auf die 40 Unternehmen des Samples bezogene Daten wurden für die Jahre 1960 bis 1999 erhoben. ${ }^{7}$ Insgesamt ergeben sich 1600 Beobachtungspunkte (40 Unternehmen mal 40 Jahre). Weil einige der betrachteten Unternehmen erst nach 1960 gegründet wurden, für manche Unternehmen und Zeitpunkte keine Informationen zur Verfügung standen, zum Erhebungszeitpunkt nicht beendete Amtszeiten nicht gewertet wurden und einige Unternehmen lange Zeit Kollektivgremien ohne Vorstandsvorsitzende oder -sprecher hatten, ${ }^{8}$ reduziert sich die Zahl der validen Beobachtungspunkte auf ungerundet genau 1000. Die längste erhobene Amtszeit ist der 25 Jahre andauernde Vorstandsvorsitz von Georg W. Claussen bei Beiersdorf. In den neunziger Jahren nahm die Bereitschaft der Aufsichtsratsgremien, Verträge von Vorstandsvorsitzenden nicht zu verlängern oder vorzeitig aufzulösen, deutlich zu. Der formalen Auflösung der Verträge griffen in die Kritik geratene Vorstandsvorsitzende in einigen Fällen durch Rücktritt vorweg.

5) In sieben Fällen - Hans U. Brauner, Michael Frenzel, Jörg Kuchenwald, Eberhard von Kuenheim, Heinz Ruhnau, Wilhelm Simson und Klaus Wiegandt- wurden die Karriereverläufe als Mischtypen klassifiziert.

6) Die längste im Sample vertretene Hauskarriere hatte Hermann Josef Strenger hinter sich, der bei Bayer ab 1949 eine kaufmännische Ausbildung absolvierte und 1983 Vorsitzender des Vorstands wurde. Nach dem Vorstandsvorsitz wechselte Strenger in den Aufsichtsrat und hat damit bereits mehr als ein halbes Jahrhundert bei Bayer verbracht.

7) Für diese Recherchen konnte das Geschäftsberichtsarchiv von Prof. Dieter Sadowski an der Universität Trier genutzt werden. Insgesamt wurden um die 500 Geschäftsberichte eingesehen.

8) Das deutsche Aktienrecht schreibt die Wahl oder Ernennung eines Vorstandsvorsitzenden nicht zwingend vor (\$84 AktG). Stärker als amerikanische Führungsgremien verstehen sich deutsche Vorstände als Kollektivgremien, in denen gemeinsam entschieden wird. Bei RWE wurde bis 1989 auf einen Vorstandsvorsitzenden verzichtet. Auch bei Agiv, AVA, Holzmann, Karstadt, Schering, Südzucker und Viag gab es bis in die achtziger Jahre hinein keinen Vorstandsvorsitzenden. Die Deutsche Bank hat bis heute keinen Vorstandsvorsitzenden, sondern einen Vorstandssprecher, der nicht vom Aufsichtsrat gewählt, sondern vom Vorstand ernannt wird. 


\section{Schaubild 3: Durchschnittliche Amtszeiten von Vorstandsvorsitzenden, 40 Unternehmen, 1960-1997}

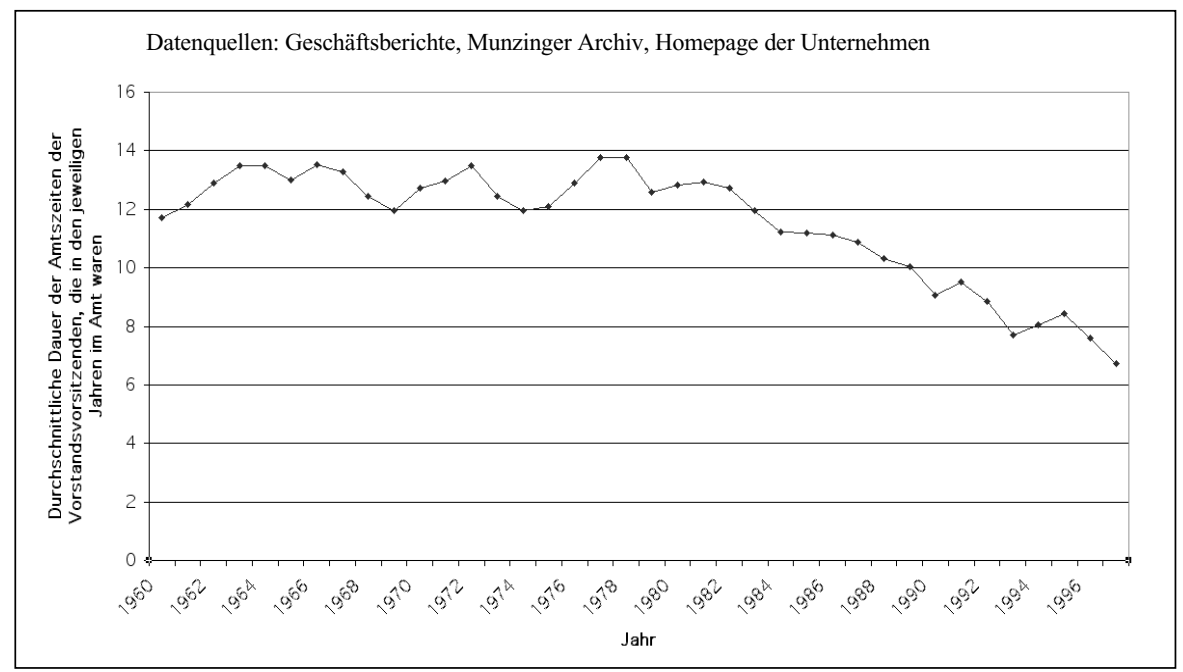

In Schaubild 3 wurde Jahr für Jahr abgetragen, wie lange die jeweils amtierenden Vorstandsvorsitzenden insgesamt im Amt waren. Anschließend wurden Jahresdurchschnitte errechnet. Wohlgemerkt wurden nur Amtszeiten berücksichtigt, die bereits beendet waren. Für das Jahr 1997 konnten 20 Amtszeiten berücksichtigt werden, von denen bei Abschluss der Recherchen bereits bekannt war, wie lange sie insgesamt andauerten. Die Kurve hat also keine »natürliche«, aus der Berechnungsweise resultierende Tendenz, im späteren Betrachtungszeitraum zu fallen. Es zeigt sich, dass für die sechziger und siebziger Jahre durchschnittliche Amtszeiten von um die 13 Jahren typisch waren. Ab etwa 1979 setzte ein Trend zu steigenden Fluktuationsraten ein. Die durchschnittliche Verweildauer der Vorstandsvorsitzenden in ihren Ämtern sank bis auf etwa sieben Jahre zum Zeitpunkt 1997. Auch dieser Befund indiziert rapiden Wandel.

Die Auswertung der erhobenen Managerbiographien zeigt, dass in den neunziger Jahren Wandel in mehrerlei Hinsicht stattfand. In den späten Neunzigern gab es unter den Vorstandsvorsitzenden der 40 Größten keine Nichtstudierten mehr. Unter den Vorstandsvorsitzenden befanden sich zu diesem Zeitpunkt mehr studierte Wirtschaftswissenschaftler als Techniker und Naturwissenschaftler. Gleichzeitig stieg der Anteil der Vorstandsvorsitzenden, die berufliche Erfahrungen in Finanzressorts gemacht hatten. Die Bedeutung des unternehmensexternen Arbeitsmarkts für Führungskräfte nahm in den neunziger Jahren deutlich zu, während der Anteil der Hauskarrieren zurückging. Die durchschnittliche Amtsdauer der Vorstandsvorsitzenden sank rapide.

In der soziologischen Managementforschung ist argumentiert worden, dass typische Erfahrungsmuster von Managern neben fachlichen Kompetenzen auch deren Wertmuster, Einstellungen und - mittelbar - auch deren Entscheidungen beeinflussen. Die veränderten Hintergründe deutscher Führungskräfte lassen sich mit einer Professionalisierung, einer Stärkung der betriebswirtschaftlichen Basis und einer zunehmenden Vermarktlichung umschreiben. Die zunehmende Vermarktlichung zeigt sich im Bedeutungszuwachs der Arbeitsmärkte für Führungskräfte und den kürzer werdenden Amtsdauern. Im Ergebnis nahm der Wettbewerb und damit die kompetitive Orientierung der Topmanager zu. 
Diese Beobachtungen helfen zu verstehen, warum in den neunziger Jahren eine grundsätzliche Bereitschaft zur Übernahme angloamerikanischer Managementkonzepte bestand und warum die Anstöße für investorenorientierte Unternehmenspolitik auf fruchtbaren Boden fielen. Vorstandschefs unterschiedlicher Industriezweige wären in den sechziger Jahren nicht auf den Gedanken gekommen, miteinander in Konkurrenz zu stehen. Jeder war Fachmann auf seinem Gebiet. Aktienkurse waren Nebensache, und durch das Vorherrschen von Hauskarrieren kamen sich Karrierewege gegenseitig nicht in die Quere. Das begünstigte die Entstehung und Festigung von langfristigen, kooperativen, netzwerkartigen Beziehungen zwischen Großunternehmen. Die Vermarktlichung der Führungskräfterekrutierung setzte Konkurrenz zunehmend an die Stelle von Kooperation. Es ist kein Zufall, dass der Durchbruch »objektiver« Rentabilitätskennzahlen und der Fokus auf die Börsenkurse mit der Vermarktlichung der Managerrekrutierung - bei gleichzeitig gestärkter betriebswirtschaftlicher Basis - zusammenfiel. Finanzielle Kennziffern machten die Performanz miteinander konkurrierender Führungskräfte über Branchengrenzen hinweg vergleichbar. Die neuen, der angloamerikanischen Praxis angenäherten Karrieremuster und die Übernahme von Strategien, die auf die Steigerung unternehmensübergreifender, standardisierter Größen wie den Discounted Cash-flow oder den Aktienkurs zielen, verhalten sich zueinander komplementär.

Wenn festgestellt wird, dass flexible, marktorientierte Rekrutierungs- und Karrieremuster und kapitalmarktorientierte Unternehmensführung gut zu einander passen, sagt dies noch nichts über die Richtung des Zusammenhangs aus. Letztlich liegt hier ein Fall rekursiver Kausalität vor. Denn nicht nur beeinflussen - einerseits - die Karrierehintergründe der Führungskräfte deren Orientierungen und damit, mittelbar, deren Bereitschaft oder Abneigung zur Verfolgung bestimmter Unternehmensziele. Anstöße zur Veränderung des Arbeitsmarkts für Führungskräfte kommen gleichzeitig - andererseits - von den «ungeduldiger « werdenden Aktionären, deren Präferenzen durch Shareholder-Value-Politik verstärkte Berücksichtigung finden. Worauf es hier aber ankommen soll, ist der nichtrekursive Teil des Zusammenhangs, der von den »Managervariablen« zur kapitalmarktorientierten Unternehmensführung verläuft. Professionalisierung und Vermarktlichung schaffen ein Klima, das kapitalmarktorientierte Unternehmensführung begünstigt und einer Gruppe von Führungskräften mit anderen als den traditionell »typisch deutschen « Merkmalen, insbesondere in Zusammenhang mit externen Marktmechanismen, zu Aufstieg und Status verhilft. Damit wird verständlich, warum Aktionärsorientierung bei deutschen Managern in den neunziger Jahren in hohem Ansehen stand (Schaubild 1) - und warum Führungskräfte nicht nur als Getriebene, sondern auch als Treiber des Shareholder Value auftraten.

\section{Entflechtung impliziert abnehmendes internes Monitoring}

Im Folgenden wird gezeigt, dass in den neunziger Jahren eine rückläufige Tendenz bei der Personalverflechtung zwischen deutschen Großunternehmen zu beobachten war. In Abgrenzung zu liberalen Verflechtungskritikern wird davon ausgegangen, dass Unternehmensverflechtungen nicht nur der Abschottung vor der Kontrolle durch Outsider (Kapitalmarktteilnehmer), sondern auch der effektiven Kontrolle durch Insider dienen. Dieser Befund relativiert die mit der zunehmenden Handlungsfähigkeit der Kapitalmarktteilnehmer einhergehende Begrenzung des Handlungsspielraums der Manager insofern, als dass der zunehmenden Überwachung durch Outsider gleichzeitig ein abnehmendes Monitoring durch Insider gegenübersteht. Im darauf folgenden Abschnitt wird vorgeführt, welche Auswirkungen diese Entwicklung auf die Höhe der Managergehälter hatte und warum sich dies förderlich auf die Bereitschaft der Führungskräfte auswirkte, auf externen Marktdruck mit kapitalmarktorientierter Unternehmensführung zu reagieren. 
Zur Funktion von Unternehmensverflechtungen existieren zwei konträre Positionen. Liberale Kritiker wie Michael Adams (1994, S. 148-153) betonen die Wirkungen, die von Unternehmensverflechtungen auf die Funktionsfähigkeit der Kapital- und Übernahmemärkte ausgehen. Adams charakterisiert das als »Deutschland $\mathrm{AG}$ « bezeichnete Industrienetzwerk als System von Ring- und Überkreuzverflechtungen, das mit dem Ziel der Abschottung vor dem Kapitalmarkt und insbesondere vor feindlichen Übernahmen aufrechterhalten wird. Im Ergebnis, argumentiert Adams, halten sich die Vorstände von Finanzunternehmen mit solchen Konstruktionen gegenseitig den Rücken vor unerwünschten Einmischungen durch die Kapitalmarktteilnehmer frei und verschaffen sich den Freiraum, persönliche Ziele zu verfolgen. Dieser Hypothese zufolge dienen Verflechtungen der Stabilisierung von Managerherrschaft zu Ungunsten der Kapitalmarktteilnehmer. ${ }^{9}$

Die elaboriertesten Analysen zu Unternehmensverflechtungen in Deutschland wurden von Jürgen Beyer und Paul Windolf vorgelegt (Beyer 1998; Windolf/Beyer 1995; Windolf 2002). Der Sicht liberaler Kritiker, der zufolge Ring- und Überkreuzverflechtungen auf Abschottung vor den Kapital- und Übernahmemärkten zielen, widersprechen Beyer und Windolf im Prinzip nicht. Allerdings wird eingewandt, dass solche heterarchischen Verflechtungsmuster - in deren Terminologie: Kreise und reziproke Cliquen - den Ausnahme- statt Regelfall darstellen und fast ausschließlich im Finanzsektor vorkommen. Diese Überkreuzbeteiligungen dienen in der Tat dem Übernahmeschutz. "Bei der gegebenen Struktur muss sich jemand, der ein Auge auf die Allianz oder die Münchener Rück wirft, schon sehr genau überlegen, ob er sich mit den beiden anlegen will «, ${ }^{10}$ begründete der Münchener Rück-Vorstandsvorsitzende Hans-Jürgen Schinzler die wechselseitige Kapitalbeteiligung.

Außerhalb des Finanzsektors dominieren allerdings horizontale Verflechtungsmuster (Pyramiden und Sterne), die - so Beyer und Windolf - nicht der Abschottung, sondern der Durchsetzung von Herrschaft und der Weitergabe von Informationen dienen (Beyer 1999, S. 526; Windolf/Beyer 1995, S. 4). Als weiteres Merkmal der deutschen Verflechtungsstrukturen charakterisieren Beyer und Windolf den hohen Übereinstimmungsgrad zwischen Kapital- und Personalverflechtungen. Personalverflechtungen kommen durch die Entsendung von Vorstandsmitgliedern in die Aufsichtsräte der Zielunternehmen zustande, dienen der Durchsetzung von Eigentümermacht und induzieren damit Kontrolle statt Abwesenheit von Kontrolle (Windolf/Beyer 1995, S. 18). Eine besonders starke Überwachung ist dort zu vermuten, wo das Senderunternehmen den Aufsichtsratsvorsitz des Zielunternehmens übernimmt.

Als wichtigste Senderunternehmen von Kapital- und Personalverflechtung wurden die deutschen Finanzunternehmen identifiziert (Beyer 2003). Die Kritik an Unternehmensverflechtungen wurde deshalb weitgehend in Form einer Kritik an der Macht der Banken geführt (Monopolkommission 1976). Gegen die liberale Kritik an Unternehmensverflechtungen und Bankeneinfluss und für die Sicht von Beyer und Windolf spricht, dass ein negativer Effekt von »Verflochtenheit « auf die Unternehmensperformanz im Vergleich deutscher Unternehmen nicht nachgewiesen werden konnte und sogar Studien zu überwiegen scheinen, in denen im Zweifel positive Wirkungen auf die Unternehmensperformanz festgestellt wurden. ${ }^{11} \mathrm{Zu}$ einem abweichenden Ergebnis kommt die Studie von Perlitz/Seger (1994, S. 60-64).

Entscheidend für das Argument dieses Abschnitts ist, dass die Personalverflechtung zwischen großen deutschen Unternehmen in den neunziger Jahren rückläufig war - was, folgt

9) Siehe auch Monopolkommission (1998b, S. 80); Naßmacher (1997, S. 8); Rubach/Sebora (1998, S. 177).

10) Financial Times Deutschland vom 23.2.2000, S. 1.

11) Beyer (1998, S. 101-116); Cable (1985); Monopolkommission (1998b, S. 97-98); Norton/Schmid (1996); Schmid (1996, S. 559); Thomsen/Pedersen (2000, S. 18). 
man den Darstellungen von Beyer, Windolf und anderen, ein abnehmendes internes Monitoring impliziert. Der Rückgang der Personalverflechtung geht insbesondere auf die Banken zurück, die sich vom Kreditgeschäft auf das Investmentbanking umorientieren und in diesem Zusammenhang keinen Sinn mehr in der aufwändigen Ausficht über Industrieunternehmen sehen (Beyer 2003; Höpner/Krempel 2003). Vor allem bei der Beratung im Zuge von Fusionen und Übernahmen können zu enge Beziehungen zu inländischen Industrieunternehmen zudem die Akquirierung von Aufträgen durch internationale Kunden behindern. Auch wo die Personalverflechtung bestehen bleibt, ist zu vermuten, dass sich die Art und Weise des Monitorings von Banken über Nichtbanken im Zuge der Hinwendung zum Investmentbanking und der damit einhergehenden neuen Interessenlage verändert (Monopolkommission 1998a, S. 96). Besonders deutlich wurde dies an der veränderten Haltung der Deutschen Bank zu feindlichen Übernahmen in Deutschland. War die Deutsche Bank ehedem Beschützerin vor feindlichen Übernahmen, äußerte der den Investmentbankern zuzurechnende Rolf E. Breuer sowohl während des Übernahmekampfs um Thyssen als auch nach der Mannesmann-Übernahme die Hoffnung, die jeweiligen Übernahmen würden ein »Exempel «12 setzen bzw. »Signalwirkung « ${ }^{13}$ dahingehend entfalten, dass deutsche Großbanken mittlerweile Investmentbanken wie andere auch, ohne restringierende Beziehungen zu Großunternehmen, geworden seien.

Schaubild 4: Personalverflechtungsgrade über die Entsendung von Aufsichtsratsmitgliedern, bezogen auf die 100 größten deutschen Unternehmen, 1980-1998

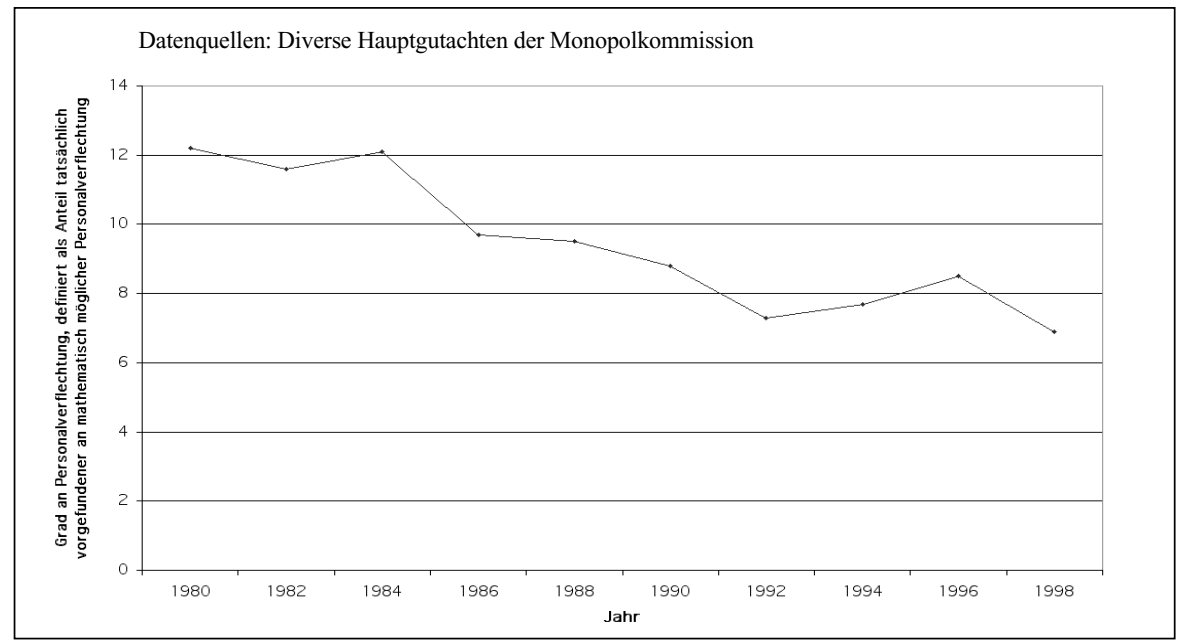

Schaubild 4 zeigt die Entwicklung des Personalverflechtungsgrads der 100 größten deutschen Unternehmen zwischen 1980 und 1998. Der Personalverflechtungsgrad wird alle zwei Jahre von der Monopolkommission errechnet und ist als Anteil der tatsächlich festzustellenden an allen mathematisch denkbaren Personalverflechtungen zwischen den 100 größten Unternehmen definiert. Das Schaubild zeigt, dass der Personalverflechtungsgrad zwischen 1980 und 1998 kontinuierlich von etwa zwölf Prozent auf um die sieben Prozent gesunken ist. Betrachtet man das (zu einem Großteil aus Finanzunternehmen bestehende) deutsche Verflech-

12) Der Spiegel 13/1997, S. 94.

13) Handelsblatt vom 28./29.1.2000, S. 8. 
tungszentrum, zeigt sich ein noch deutlicherer Wandel (siehe dazu auch die Grafiken bei Höpner/Krempel 2003). Windolf (2002) zufolge hat sich der Personalverflechtungsgrad zwischen den 15 »verflochtensten« deutschen Großunternehmen allein zwischen 1993 und 1999 etwa halbiert.

Eine abnehmende Kontrolle der Manager durch Insider geht mit dem Rückzug der Finanzunternehmen aus der Überwachung von Industrie- und Handelsunternehmen aber nur einher, wenn dieser Rückzug nicht durch andere aktive Unternehmensaufseher kompensiert wird. Wer tritt an die Stelle der Bankenvertreter? Geeignete Referenzpunkte zur Beantwortung dieser Frage sind die Vorsitze der Aufsichtsräte. In Schaubild 5 wurde abgetragen, wie viele Aufsichtsratsvorsitzende der 40 betrachteten Unternehmen zwischen 1990 und 1999 Vertreter von Finanzunternehmen waren, und wie viele von ihnen ehemalige Manager derselben Unternehmen waren und also vom Vorstand in den Aufsichtsrat wechselten. Der Anteil an Aufsichtsratsvorsitzen, der von Finanzunternehmen gestellt wurde, erweist sich bis etwa 1995 als stabil, und sinkt in den Folgejahren kontinuierlich. Der deutlichste Rückzug aus Aufsichtsratsvorsitzen ist bei der Deutschen Bank, der Vorreiterin der Umorientierung auf das Investmentbanking, zu beobachten. In ihren 2001 veröffentlichten Corporate-Governance-Grundsätzen kündigte die Deutsche Bank an, sich künftig vollständig aus den Aufsichtsratsvorsitzen von Industrie- und Handelsunternehmen zurückziehen zu wollen (Deutsche Bank 2001, S. 8).

\section{Schaubild 5: Herkunft von Aufsichtsratsvorsitzenden: Vertretern von Finanzunter- nehmen und ehemaligen Manager, 40 Unternehmen, 1990-1999}

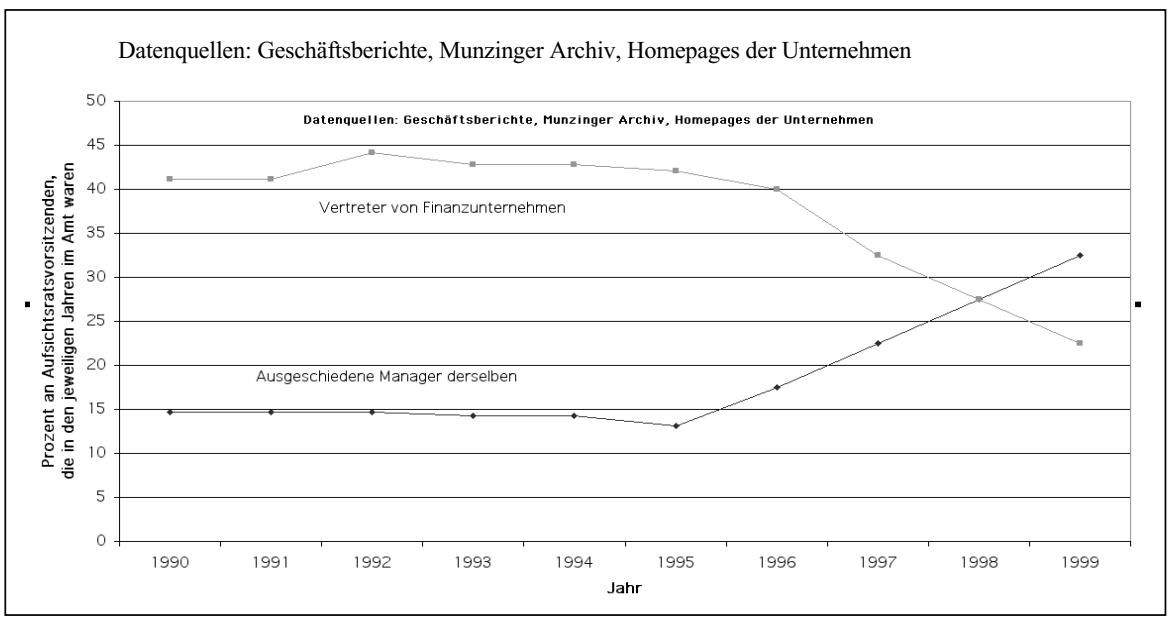

Schaubild 5 zeigt darüber hinaus, wer an die Stelle der sich zurückziehenden Bankenvertreter trat. Es waren nicht externe Experten oder etwa Vertreter von Investmentfonds, sondern ehemalige Manager derselben Unternehmen, die den Rückzug der ehemaligen Überwacher nahezu perfekt, und ebenfalls beginnend im Jahr 1995, kompensierten. Dies scheint weitgehend unbemerkt vonstatten gegangen zu sein und widerspricht auch den Empfehlungen der Regierungskommission Corporate Governance (2003, Ziffer 5.4.2.) diametral. Der zunehmenden Überwachung deutscher Unternehmensleitungen durch die Kapitalmärkte stand eine zunehmende Abschottung vor interner Kontrolle gegenüber. Aufsichtsratsvorsitze wurden zunehmend von Ex-Managern der zu überwachenden Unternehmen übernommen, was dem Gegenteil von unabhängiger Kontrolle entspricht. Das lässt die These zu, dass der Grad an 
Überwachung der Führungskräfte unter dem Strich nicht zunahm, sondern sich qualitativ veränderte.

\section{Warum die Managervergütung steigt}

Die Argumentation des vorigen Abschnitts weiterführend, wird hier gezeigt, dass Führungskräfte von Großunternehmen von dem Übergang vom internen zum externen Monitoring in doppelter Weise profitierten. Sowohl der Strategiewechsel der Banken und ihr Rückzug aus der internen Überwachung wie auch die Öffnung gegenüber der Überwachung durch die Kapitalmarktteilnehmer erhöhten die Managervergütungen. Shareholder-ValueStrategien steigerten den variablen, und die Veränderungen der internen Überwachung den fixen Vergütungsbestandteil. Das scheinbare Paradox, dass kapitalmarktorientierte Unternehmensführung bei Führungskräften in den späten neunziger Jahren trotz der damit einhergehenden Einschränkung von deren Handlungsspielräumen in hohem Kurs stand, findet damit eine zusätzliche Erklärung.

In der Agency-Literatur wie auch in deren Vorläuferin, der Literatur zur Managerherrschafts-These, wurde Führungskräften vor allem die Neigung zugesprochen, Ressourcen des Unternehmens zur Befriedigung eigener Bedürfnisse abzuschöpfen. ${ }^{14}$ Es ist evident und auf der Basis mikroökonomischer Rationalitätsannahmen geradezu tautologisch, dass Führungskräfte das Ziel hoher persönlicher Einkommen verfolgen. Deshalb verwundert es nicht, dass unternehmensvergleichende Studien zu dem Ergebnis kommen, dass die Abwesenheit von Kontrolle ausübenden Großaktionären mit höheren, und deren Anwesenheit mit niedrigeren Managergehältern einhergeht. ${ }^{15}$ Manager erhöhen ihre Einkommen, wenn fehlende interne Überwachung dies zulässt.

Die Variabilisierung der Managervergütung zählt zu den entscheidenden Instrumenten aktionärsorientierter Unternehmensführung. Prinzipiell sind zwei Wege der Variabilisierung der Managervergütung denkbar: Die Gewährung von Boni und Aktienoptionen zusätzlich zu den fixen Vergütungsbestandteilen, oder aber die Substituierung fixer Bestandteile durch variable. Die Erfahrung zeigt, dass fast immer der erste Weg gewählt wird. Das Aktienoptionsprogramm bei $B A S F$, wo Manager zur Erlangung von Aktienoptionen selbst einen Teil ihres fixen Gehalts investieren müssen, ist eine Ausnahme von der Regel. ${ }^{16}$ Daten zu den Vergütungsstrukturen zeigen, dass die Gehälter von Topmanagern trotz des Booms von Aktienoptionsprogrammen in den späten Neunzigern keinen sinkenden, sondern sogar einen deutlich steigenden Trend aufwiesen. Daraus folgt, dass Shareholder-Value-Politik grundsätzlich nicht nur mit variableren, sondern gleichzeitig mit insgesamt höheren Managergehältern einherging. Shareholder Value begrenzte durch die Öffnung für externes Monitoring nicht nur den Handlungsspielraum der Führungskräfte. Hinsichtlich der Vergütungshöhe bediente es Managerinteressen.

14) Burnham (1941, S.: 26); Jensen/Meckling (1976, S. 308); Shleifer/Vishny (1996, S. 8); Williamson (1964, S. 32). Vergleiche aber Galbraith (1967, S. 135-137), der verblüffenderweise zu dem Ergebnis kommt, dass die »Annahme, dass jedermann nach dem höchstmöglichen Einkommen strebt - dass er für sich persönlich soviel Geld wie möglich verdienen möchte - ... etwas erfrischend unsentimentales an sich « habe, aber auf die Technostruktur, die anstelle der Aktionäre die Unternehmen beherrsche, nicht zutreffe. Deren Ziele seien nicht primär ökonomisch. Zudem: »Die Technostruktur lässt die Abgeschiedenheit nicht zu, in der allein Unehrenhaftigkeit und Übeltat gedeihen.«

15) Boyd (1994, S. 340); Denis/Denis/Sarin (1997); Kraft/Niederprüm (1999, S. 24); Schmid (1997, S. 77).

16) Siehe zum BASF-Aktienoptionsprogramm Handelsblatt vom 8./9.10.1999. 
Tabelle 1: Die Entwicklung der Vorstandsvergütungen großer deutscher Unternehmen zwischen 1996 und 1999

\begin{tabular}{|c|c|c|c|c|c|c|c|}
\hline Unternehmen & $\begin{array}{c}\text { Ver- } \\
\text { gütung } \\
\text { des } \\
\text { Gesamt- } \\
\text { vor- } \\
\text { stands in } \\
\text { Mio. } \\
\text { EUR } \\
\text { (1996) }\end{array}$ & $\begin{array}{l}\text { Vor- } \\
\text { stands- } \\
\text { größe } \\
(1996)\end{array}$ & $\begin{array}{c}\text { Ver- } \\
\text { gütung } \\
\text { des } \\
\text { Gesamt- } \\
\text { vor- } \\
\text { stands in } \\
\text { Mio. } \\
\text { EUR } \\
\text { (1999) }\end{array}$ & $\begin{array}{c}\text { Vor- } \\
\text { stands- } \\
\text { größe } \\
\text { (1999) }\end{array}$ & $\begin{array}{l}\text { Durch- } \\
\text { schnitt- } \\
\text { liche } \\
\text { Ver- } \\
\text { gütung } \\
\text { pro Vor- } \\
\text { stands- } \\
\text { mitglied } \\
\text { in Mio. } \\
\text { EUR } \\
(1996)\end{array}$ & $\begin{array}{l}\text { Durch- } \\
\text { schnitt- } \\
\text { liche } \\
\text { Ver- } \\
\text { gütung } \\
\text { pro Vor- } \\
\text { stands- } \\
\text { mitglied } \\
\text { in Mio. } \\
\text { EUR } \\
\text { (1999) }\end{array}$ & $\begin{array}{c}\text { Wachs- } \\
\text { tum der } \\
\text { Ver- } \\
\text { gütung } \\
\text { pro Vor- } \\
\text { stands- } \\
\text { mitglied } \\
\text { in } \\
\text { Prozent } \\
\text { (1996- } \\
1999)\end{array}$ \\
\hline Agiv AG & 1,21 & 3 & 6,75 & 5 & 0,40 & 1,35 & 235,7 \\
\hline AVA AG & 0,84 & 3 & 4,00 & 3 & 0,28 & 1,33 & 374,4 \\
\hline Axel Springer Verlag AG & 3,05 & 4 & 6,32 & 6 & 0,76 & 1,05 & 38,3 \\
\hline BASF AG & 9,82 & 11 & 9,22 & 9 & 0,89 & 1,02 & 14,8 \\
\hline Bayer AG & 7,68 & 8 & 8,24 & 8 & 0,96 & 1,03 & 7,3 \\
\hline Beiersdorf AG & 2,96 & 6 & 3,71 & 6 & 0,50 & 0,62 & 25,3 \\
\hline Bilfinger+Berger AG & 3,93 & 7 & 2,51 & 4 & 0,56 & 0,63 & 11,8 \\
\hline BMW AG & 7,06 & 7 & 7,22 & 8 & 1,01 & 0,90 & $-10,5$ \\
\hline Buderus AG & 1,36 & 4 & 1,79 & 4 & 0,34 & 0,45 & 32,0 \\
\hline Continental AG & 4,28 & 5 & 5,20 & 6 & 0,85 & 0,86 & 1,2 \\
\hline Daimler-Benz AG & 7,16 & 10 & 52,72 & 13 & 0,72 & 4,06 & 466,5 \\
\hline Degussa AG & 4,45 & 6 & 9,22 & 11 & 0,74 & 0,84 & 13,1 \\
\hline Deutsche Babcock AG & 1,64 & 3 & 3,76 & 3 & 0,55 & 1,25 & 129,1 \\
\hline Deutsche Lufthansa AG & 2,20 & 6 & 2,10 & 4 & 0,37 & 0,53 & 42,9 \\
\hline Deutsche Telekom AG & 5,29 & 8 & 7,60 & 8 & 0,66 & 0,95 & 43,6 \\
\hline Deutz AG & 1,03 & 3 & 1,62 & 4 & 0,34 & 0,40 & 18,5 \\
\hline Henkel KGaA & 6,66 & 9 & 8,58 & 8 & 0,74 & 1,07 & 44,8 \\
\hline Hoechst AG & 7,04 & 9 & - & 8 & 0,78 & - & - \\
\hline Holzmann AG & 1,78 & 6 & 2,84 & 5 & 0,30 & 0,57 & 91,1 \\
\hline Karstadt AG & 4,44 & 7 & 6,53 & 7 & 0,63 & 0,93 & 46,8 \\
\hline Krupp AG & 3,99 & 5 & 9,02 & 9 & 0,80 & - & $25,6^{1)}$ \\
\hline Linde AG & 4,29 & 7 & 3,81 & 6 & 0,61 & 0,63 & 3,82 \\
\hline MAN AG & 4,23 & 8 & 4,82 & 8 & 0,53 & 0,6 & 14,1 \\
\hline Mannesmann AG & 5,72 & 7 & 10,04 & 6 & 0,82 & 1,67 & 105 \\
\hline Metallgesellschaft AG & 4,14 & 5 & 6,12 & 5 & 0,83 & 1,22 & 47,6 \\
\hline Metro AG & 7,98 & 6 & 6,06 & 5 & 1,33 & 1,21 & $-8,9$ \\
\hline Preussag AG & 4,73 & 8 & 5,16 & 6 & 0,59 & 0,86 & 45,7 \\
\hline Rheinmetall AG & 0,86 & 6 & 1,25 & 5 & 0,14 & 0,25 & 75,2 \\
\hline RWE AG & 3,95 & 11 & 3,75 & 5 & 0,36 & 0,75 & 108,5 \\
\hline SAP AG & 3,84 & 8 & 5,54 & 5 & 0,48 & 1,11 & 130,9 \\
\hline Schering AG & 3,88 & 5 & 6,57 & 5 & 0,78 & 1,31 & 69,6 \\
\hline Siemens AG & 10,48 & 13 & 21,75 & 11 & 0,81 & 1,98 & 145,2 \\
\hline Spar AG & 1,39 & 3 & 1,10 & 3 & 0,47 & 0,37 & $-21,1$ \\
\hline Strabag AG & 1,96 & 6 & 1,46 & 3 & 0,33 & 0,49 & 48,9 \\
\hline Südzucker AG & 1,92 & 5 & 2,20 & 5 & 0,38 & 0,44 & 15,0 \\
\hline Thyssen AG & 5,88 & 7 & 9,02 & 9 & 0,84 & - & $19,3^{1)}$ \\
\hline VEBA AG & 8,64 & 9 & 9,62 & 9 & 0,96 & 1,07 & 11,3 \\
\hline VEW AG & 1,66 & 3 & 2,52 & 4 & 0,55 & 0,63 & 13,7 \\
\hline Viag AG & 2,81 & 4 & 3,58 & 5 & 0,70 & 0,72 & 2,1 \\
\hline Volkswagen AG & 6,76 & 7 & 8,60 & 5 & 0,97 & 1,72 & 78,2 \\
\hline
\end{tabular}


Nachfolgend werden die fixen Vergütungsbestandteile näher betrachtet. Tabelle 1 informiert über die Entwicklung der Managergehälter zwischen 1996 und 1999. Diese Angaben stammen aus Geschäftsberichten. Aus den dort angegebenen Beträgen sowie der Größe der Vorstände konnte das Durchschnittsgehalt eines Vorstandsmitglieds errechnet werden. Diese Angaben sind insofern kontrafaktisch, als dass die Gehälter der Mitglieder ein- und desselben Vorstands in Wahrheit nicht gleich sind und insbesondere der Vorstandsvorsitzende höhere Bezüge erhält als seine Vorstandskollegen. Das durchschnittliche Jahresgehalt des Vorstandsmitglieds eines durchschnittlichen Unternehmens aus dem Kreis der 40 größten börsennotierten Aktiengesellschaften lag demnach im Jahr 1996 bei 640.000 Euro, wobei sich hinter diesem Durchschnitt eine hohe Varianz verbirgt. Das niedrigste Vorstandsgehalt gab es bei Rheinmetall, wo 143.000 Euro gezahlt wurden. Spitzenreiter war Metro mit 1,3 Millionen Euro. Damit unterschieden sich niedrigstes und höchstes Gehalt im betrachteten Sample um den Faktor 9,3, mit einer Spannweite von 1,14 Millionen Euro.

Sowohl für amerikanische (Conyon/Leech 1994, S. 239) als auch für deutsche Unternehmen (Backes-Gellner/Geil 1997, S. 470; Kraft/Niederprüm 1999, S. 24; Schmid 1997, S. 79) wurde ein positiver Zusammenhang zwischen Vorstandsvergütung und Unternehmensgröße festgestellt. Dieser Zusammenhang zeigt sich auch bei den hier betrachteten Daten. Sowohl die Höhe der Vorstandsvergütungen im Ausgangsjahr 1996 ( $r=.37, p=.018, n=40)$ als auch die Steigerungsraten zwischen 1996 und 1999 (r=.32, p=.048, n=39) sind positiv mit der Unternehmensgröße, gemessen anhand der Wertschöpfung im Jahr 1996, korreliert. Aussagen über Bestimmungsgründe der Entwicklung der Vorstandsgehälter sind deshalb nur unter statistischer Kontrolle für die Unternehmensgröße sinnvoll.

Es zeigen sich zwei Determinanten der Vorstandsvergütungen im Jahr 1996, die sich auf die Stärke bzw. die Schwäche beziehen, mit der Führungskräfte intern überwacht werden. Eine schwache Aufsicht liegt vor, wenn der Aufsichtsratsvorsitzende ein ehemaliger Manager desselben Unternehmens ist. In Abschnitt 3 wurde gezeigt, dass diese Form der Abwesenheit von Kontrolle in der zweiten Hälfte der neunziger Jahre zunahm. Eine Variable wurde gebildet, indem für alle 40 Unternehmen die Anzahl der Jahre zwischen 1996 und 1999 gezählt wurde, in denen jemand den Aufsichtsratsvorsitz innehatte, der aus demselben Unternehmen stammte. Sowohl in der bivariaten Korrelation $(r=.34, p=.037, n=39)$ als auch in der für die Unternehmensgröße kontrollierenden Regression zeigen sich signifikant positive Zusammenhänge zwischen der Höhe der Vergütungen im Jahr 1996 und der Variable, die Managerherrschaft im Sinne der Abwesenheit unabhängiger interner Kontrolle abbildet (Gleichung 1 im Anhang). Der Schätzgleichung zufolge liegt das Vorstandsjahresgehalt in einem managerkontrollierten Unternehmen im Vergleich zu einem nicht managerkontrollierten Unternehmen bei gleicher Unternehmensgröße um durchschnittlich etwa 180.000 Euro höher - was immerhin ein höherer Betrag ist als das durchschnittliche Jahresgehalt eines Vorstandsmitglieds von Rheinmetall, und immerhin ein Viertel der durchschnittlichen Jahresvergütung bei den Unternehmen des untersuchten Samples. Dieses Ergebnis stützt die Annahme von Managerherrschafts- und Agency-Theorie, dass Manager unkontrollierte Freiräume nutzen, um persönliche Interessen zu verfolgen.

Spiegelbildlich dazu fiel die Vorstandsvergütung niedriger aus, wenn der Aufsichtsratsvorsitzende ein Vertreter einer Bank war. Ein (wenn auch schwach) signifikantes Ergebnis kommt sowohl für die bivariate Korrelation $(r=.-27, p=.083, n=40)$ als auch für die Regression unter statistischer Kontrolle für die Unternehmensgröße ${ }^{17}$ (Gleichung 2 im Anhang) zu-

17) Wenn der Aufsichtsratsvorsitzende in den vier Jahren zwischen 1996 und 1999 ein Bankenvertreter war, lag die durchschnittliche Vorstandsvergütung demnach um etwa 120.000 Euro niedriger, verglichen mit einer Situation, in der kein Bankenvertreter Aufsichtsratsvorsitzender war. 
stande. Dieses Ergebnis stützt die im vorigen Abschnitt postulierte Annahme, dass Bankenvertreter Unternehmensvorstände tatsächlich effektiv intern kontrollieren. Für Verflechtungskritiker wie Adams (1994) indizieren Personalverflechtungen mit Finanzunternehmen gerade das Gegenteil von Kontrolle, nämlich die Absicherung von Managerherrschaft. Kontrolle durch Bankenvertreter und »Kontrolle « durch ehemalige Kollegen wirken in der Frage der Managervergütungen, wie diese Ergebnisse zeigen, in unterschiedliche Richtungen. Die Interpretation der Funktionalität von Unternehmensnetzwerken vor allem bei Beyer und Windolf gewinnt im Licht der hier ausgewerteten Daten an Plausibilität. Allerdings fanden in den späten neunziger Jahren sowohl ein Rückzug der Banken aus Aufsichtsratsgremien und insbesondere deren Vorsitzen als auch eine grundsätzliche Veränderung der Interessenslage, die Großbanken gegenüber Industrieunternehmen einnehmen, statt.

Für die Vorstandsbezüge im Jahr 1999 wurden analoge Berechnungen durchgeführt. Aus den Daten für 1996 und 1999 ließen sich die prozentualen Wachstumsraten der Vorstandsgehälter in den späten neunziger Jahren errechnen, die in der letzten Spalte der Tabelle 1 wiedergegeben werden. An den Steigerungsraten verblüfft deren zum Teil enorme Höhe. Im Schnitt stiegen die Vorstandsbezüge in nur drei Jahren um nominal (nicht inflationsbereinigt) 66 Prozent, verglichen mit Lohnerhöhungen der tariflich Beschäftigten, die nominal weniger als zehn Prozent betrugen. Wohlgemerkt: Aktienoptionsprogramme sind bei diesen Steigerungsraten nicht eingerechnet. Die Steigerungsraten der effektiven Gesamtvergütungen liegen deshalb wesentlich höher.

Noch auffälliger ist allerdings die Unterschiedlichkeit der nominalen Steigerungsraten. In den Handelsunternehmen Spar - nomen est omen - sowie Metro und bei BMW sind die Vorstandsbezüge sogar gesunken. Bei Unternehmen wie Continental, Bilfinger+Berger, Bayer, Degussa, MAN, VEW und Veba fielen die Gehaltserhöhungen moderat und vergleichbar mit den Lohnerhöhungen der abhängig Beschäftigten aus. Ausreißer nach oben war DaimlerChrysler, wo die Vorstandsgehälter um den Faktor 4,6 stiegen. Ein Vorstandsmitglied bei DaimlerChrysler bezog 1999 ein durchschnittliches Festgehalt von 3,8 Millionen Euro. Dieser Sonderfall ist mit der Fusion zwischen Daimler-Benz und Chrysler zu erklären. Aktionärsaktivisten wie Ekkehard Wenger warfen dem Management von DaimlerBenz in diesem Zusammenhang vor, der schwierige Zustand von Chrysler sei bereits vor der Fusion bekannt gewesen, und die Motivation zur Fusion habe von der Möglichkeit hergerührt, die deutschen Vorstandsgehälter auf amerikanisches Niveau anheben zu können. ${ }^{18}$ Auch bei Agiv erfolgte zwischen 1996 und 1999 eine Erhöhung der Bezüge um mehr als 100 Prozent, was ebenfalls als Sonderfall erklärbar ist: Da der Konzern kurz vor der Auflösung stand, konnte das Management für Selbstbedienungspraktiken nicht mehr abgestraft werden. ${ }^{19}$ Auch dieses Beispiel zeigt, dass das Ziel der Einkommenssteigerung von Managern tatsächlich verfolgt wird, wenn deren Freiräume es zulassen. Tabelle 1 zeigt hohe Steigerungsraten auch bei Mannesmann, RWE, Siemens und SAP.

Als Bremser der Erhöhung der Managergehälter erweist sich die Präsenz hauptamtlicher Gewerkschafter auf den Arbeitnehmerbänken der Aufsichtsräte. ${ }^{20}$ Es wurde der Prozentsatz an Aufsichtsratsmandaten ermittelt, der von unternehmensexternen Gewerkschaftsvertretern

18) So Ekkehard Wenger im Interview mit dem Handelsblatt (vom 29.11.2000, S. 16) unter dem Titel »Chrysler ist ein Schrottladen«.

19) Auf der Hauptversammlung im Juni 2000 drohte ein Eklat, als die gestiegenen Vergütungen zur Sprache kamen; Aktionärsaktivisten reagierten mit heftigen Attacken, bezeichneten die Vorgänge als »skandalös und ungeheuerlich « und die Vergütungssteigerungen als »Schlachtfestprämie«. Neben den in Tabelle 1 ersichtlichen Mehrverdiensten für Vorstandsmitglieder stiegen die Aufsichtsratsbezüge von 970.000 Euro auf 3,2 Millionen (!) Euro. Siehe Handelsblatt vom 28.6.2000, S. 16.

20) Diese Daten wurden von der Monopolkommission freundlicherweise zur Verfügung gestellt. 
eingenommen wurde (Datenquelle: Monopolkommission). Dieser Prozentsatz und die Steigerungsraten der Managervergütung sind negativ korreliert $(r=-34, p=.033, n=39)$. Ein signifikant bremsender Effekt externer Arbeitnehmervertreter zeigt sich auch in der Regression unter statistischer Kontrolle für die Unternehmensgröße (Gleichung 3 im Anhang). Demnach mindert jeder Externe auf der Arbeitnehmerbank ${ }^{21}$ eines aus 20 Mitgliedern bestehenden Aufsichtsrats die (insgesamt zwischen $-21,1$ und $+466,5$ Prozentpunkte streuende) Steigerung der Vorstandsvergütungen zwischen 1996 und 1999 um beachtliche 50 Prozentpunkte. Einen die Höhe der Managervergütungen begrenzenden Effekt der Arbeitnehmermitbestimmung hatte bereits Frank A. Schmid (1997, S. 79) in seiner Analyse der Vorstandsbezüge von 110 großen deutschen Aktiengesellschaften herausgestellt. Das hier gefundene Ergebnis legt den Schluss nahe, dass hauptamtliche Gewerkschafter eher als interne Arbeitnehmervertreter gewillt sind, Selbstbedienungspraktiken von Führungskräften Grenzen zu setzen. Das offensichtliche Fehlverhalten des ehemaligen IG Metall-Vorsitzenden Klaus Zwickel im Zuge der Bewilligung der abnormal hohen Abfindungen im Fall Mannesmann, in den Jahren 2003 und 2004 strafgerichtlich geprüft (zum Zeitpunkt der Abfassung dieses Beitrags noch andauernd), sollte deshalb nicht vorschnell verallgemeinert werden. Gerade eine Entfernung der unternehmensexternen Gewerkschaftsfunktionäre aus den Aufsichtsräten, von Mitbestimmungskritikern immer wieder vehement gefordert, ${ }^{22}$ könnte Kontrollverluste nach sich ziehen, die Effekte des Rückzugs der Bankenvertreter noch vergrößern und im Endeffekt zu steigenden Agency-Kosten führen.

Für das Jahr 1996 zeigte sich ein deutlich dämpfender Effekt des Monitorings durch Banken auf die Höhe der Vorstandsvergütungen. Zwischen 1996 und 1999 haben in bankenüberwachten Unternehmen überdurchschnittliche Erhöhungen stattgefunden. Das zeigt sowohl die bivariate Korrelation ( $\mathrm{r}=.36, \mathrm{p}=.023, \mathrm{n}=39$ ) als auch die Regression, bei der der Größeneffekt herausgerechnet wird (Gleichung 4 im Anhang). Insgesamt erklären die Unternehmensgröße, das Monitoring durch externe Gewerkschafter und die Übernahme der Aufsichtsratsvorsitze durch Bankenvertreter 44 Prozent der Gesamtvarianz (Gleichung 5 im Anhang). Hinsichtlich der Banken sind also zwei Vorgänge zu bedenken. Erstens: Der dämpfende Effekt auf die Vorstandsvergütungen, der von Bankenvertretern als Aufsichtsratsvorsitzenden ausging, schwächte sich in den späten Neunzigern merklich ab. Die von Bankenvertretern überwachten Unternehmen machten ihren noch 1996 deutlichen Rückstand bei den Managervergütungen zum Teil wieder wett. ${ }^{23}$ Das deutet auf eine Veränderung des Monitorings durch Banken im Übergang von Haus- zu Investmentbanken hin. ${ }^{24}$ Zweitens: Bankenvertreter zogen sich aus Aufsichtsräten, besonders aus Aufsichtsratsvorsitzen, zurück. Damit ist nachgewiesen, dass die Führungskräfte hinsichtlich ihres Gehalts vom partiellen Übergang von der internen zur externen Unternehmensüberwachung profitieren.

21) Die Zahl der von Gewerkschaftsvertretern wahrgenommenen Aufsichtsratsmandate streut im betrachteten Sample von Null (Axel Springer Verlag als Tendenzbetrieb, SAP als quasi gewerkschaftsfreies Unternehmen) bis vier (Thyssen, Mannesmann) in den damals montanmitbestimmten Unternehmen.

22) Ausführlich zur Mitbestimmungsdebatte: Höpner (2004).

23) In den Vergütungsstrukturen von 1999 zeigt sich unter statistischer Kontrolle für die Unternehmensgröße nur noch ein schwach bremsender Effekt des Monitorings durch Bankenvertreter (siehe Gleichung 6 im Anhang).

24) Auch und gerade bei den Großbanken fanden in den späten Neunzigern erhebliche Steigerungen der Managervergütungen statt. Neben der veränderten Interessenlage der Banken ist dies ein Grund für den beschriebenen Effekt. Initiativen zu Deckelungen von Vergütungssteigerungen durch Bankenvertreter als Aufsichtsratsvorsitzende wären unglaubwürdig gewesen. 


\section{Schluss}

Auf Grundlage einer Verknüpfung von ökonomischer Theorie und Soziologie des Managements wurden in diesem Beitrag die Motive der Führungskräfte bei Einführung von Strategien aktionsärsorientierter Unternehmensführung diskutiert. Voraussagen der Agency-Theorie lassen vermuten, dass externer Marktdruck vonnöten ist, um Führungskräfte zu aktionärsorientiertem Handeln anzuhalten. Empirische Studien bestätigen die Erwartungen der AgencyTheorie, die aber keine Aussagen über die sozialen Prozesse hinter der Umsetzung solcher Strategien enthält. Es zeigt sich, dass Shareholder-Value-Strategien nicht gegen den Willen der Führungskräfte durchgesetzt werden müssen - sondern dass es spezifischen Gruppen von Führungskräften gelingt, die Agency-Mechanismen zur Erhöhung ihres Status, ihrer Aufstiegschancen und ihrer Einkommen zu nutzen. Vor diesem Hintergrund schwenken auch Manager »alten Typs« auf Strategien aktionärsorientierter Unternehmensführung um. So kommt ein Diffusionsprozess von Shareholder-Value-Strategien in Gang, in dem aktionärsorientierte Unternehmensführung als Managementpraktik positives Ansehen genießt, obwohl sie den unkontrollierten Handlungsspielraum der Führungskräfte auf den ersten Blick einschränkt.

Allerdings lässt sich die Frage, ob Manager durch die sich verändernden Konstellationen gewinnen oder verlieren, nicht pauschal, sondern nur im Hinblick auf die jeweiligen Felder der Unternehmenspolitik beantworten. Die Agency-Theorie hat herausgestellt, dass Manager eine höhere Risikoaversion als Investoren und deshalb eine Präferenz für die Diversifizierung der Unternehmensaktivitäten über unterschiedliche Geschäftsfelder hinweg haben. Dieselbe Präferenz haben Kreditgeber. Als das Monitoring über Unternehmen zu einem großen Teil von Banken geleistet wurde und diese das Kreditgeschäft als Kernaktivität definierten, wurde Managern bei der Streuung der Geschäftsbereiche relativ große Freiräume gelassen. Der Handlungsspielraum der Führungskräfte wird in dieser Frage durch die zunehmende Handlungsfähigkeit der Anteilseigner begrenzt. Rückläufig ist auch der Freiraum für Manager, im Interesse des Unternehmenswachstums unrentable Unternehmensteile (im Kerngeschäftsfeld oder der Peripherie) dauerhaft durch Quersubventionierung im Unternehmen zu halten. Sowohl die Zunahme des internationalen Wettbewerbs als auch Veränderungen auf den Aktienmärkten schränken den Handlungsspielraum der Manager dahingehend ein, dass die Entwicklung der Marktkapitalisierung der Unternehmen und damit die Aktionärsrendite bei Investitionsentscheidungen zunehmend berücksichtigt werden muss.

Gleichwohl ist die Vorstellung, diese Entwicklungen fänden grundsätzlich gegen den Willen und Widerstand der Führungskräfte statt, zurückzuweisen. Wenn berichtet wird, wie deutsche Konzernlenker vor mächtigen Fondsmanagern zittern, die von ihnen kapitalmarktorientierte Unternehmensführung erwarten (Balzer/Nölting 1997, S. 81-82), deutet dies in die falsche Richtung - oder ist doch zumindest nur eine Hälfte der Wahrheit. In dem Teil des Agency-Konflikts, der das Interesse der Manager am direktesten berührt, nämlich ihrem eignen Einkommen, wird ihr Handlungsspielraum nicht enger, sondern weiter. Durch den Übergang vom internen zum externen Monitoring gewinnen Manager doppelt. Die Personalentflechtung und der Strategiewechsel der Banken steigern den fixen, und Shareholder-ValueStrategien den variablen Bestandteil der Managervergütung. Das zunehmende externe Monitoring über den Aktienkurs scheint, ebenso wie Aktionärsaktivismus auf Hauptversammlungen, in dieser Frage nichts auszurichten. Der unkontrollierte Handlungsspielraum der Führungskräfte hat sich verlagert, nicht aufgelöst. Das Ende der Managerherrschaft ist noch nicht in Sicht. 


\section{Literaturverzeichnis}

Achleitner, Ann-Christin/Bassen, Alexander (2000): Entwicklungsstand des Shareholder-Value-Konzepts in Deutschland - Empirische Befunde, Oestrich-Winkel: EBS Finance Group Working Paper 0002, February 2000.

Adams, Michael (1994): Die Usurpation von Aktionärsbefugnissen mittels Ringverflechtung in der >Deutschland AG<. Vorschläge für Reformen im Wettbewerbs-, Steuer- und Unternehmensrecht, in: Die Aktiengesellschaft 4, S. 148-158.

Backes-Gellner, Uschi/Geil, Linus (1997): Managervergütung und Unternehmenserfolg - Stand der theoretischen und empirischen Forschung, in: WISU 26, S. 468-475.

Balzer, Arno/Nölting, Andreas (1997): Die schiere Macht, in: Manager Magazin 27/8, S. 73-89.

Beyer, Jürgen (1998): Managerherrschaft in Deutschland? >Corporate Governance< unter Verflechtungsbedingungen, Opladen: Westdeutsche Verlag.

Beyer, Jürgen (1999): Unternehmensverflechtungen und Managerherrschaft in Deutschland, in: Leviathan 27, S. 518-536.

Beyer, Jürgen (2003): Deutschland AG a.D.: Deutsche Bank, Allianz und das Verflechtungszentrum des deutschen Kapitalismus. In: Wolfgang Streeck/Martin Höpner (Hrsg.), Alle Macht dem Markt? Fallstudien zur Abwicklung der Deutschland AG, Frankfurt: Campus, S. 118-146.

Bleicher, Knut (1983): Organisationskulturen und Führungsphilosophien im Wettbewerb, in: Zeitschrift für betriebswirtschaftliche Forschung 35, S. 135-146.

Boyd, Brian K. (1994): Board Control and CEO Compensation, in: Strategic Management Journal 15, S. 335-344.

Burnham, James (1941): The Managerial Revolution. What is happening in the World, Westport, Connecticut: Greenwood Press.

Byrkjeflot, Haldor (2002): Management Models and Technical Education Systems: germany and the United States 1870-1930. In: Lars Engwall/Kerstin Sahlin-Anderson (Hrsg.). The Expansion of Management Knowledge: Careers, Ideas and Sources, Palo Alto: Stanford University Press, S. 212-245.

Cable, John (1985): Capital Market Information and Industrial Performance: The Role of West German Banks, in: Economic Journal 95, S. 118-132.

Conyon, Martin J./Leech, Dennis (1994): Top Pay, Company Performance and Corporate Governance, in: Oxford Bulletin of Economics and Statistics 56, S. 229-247.

Denis, Daniel J./Denis, Diane K./Sarin, Atulya (1997): Ownership Structure and Top Executive Turnover, in: Journal of Financial Economics 45, S. 193-221.

Deutsche Bank (2001): Die Corporate Governance-Grundsätze der Deutschen Bank. Corporate Governance als Teil des Selbstverständnisses der Deutschen Bank, Frankfurt: Deutsche Bank.

Eckert, Stefan (2004): Aktionärsorientierung der Unternehmenspolitik? Shareholder Value - Globalisierung - Internationalität, Wiesbaden: Gabler.

Egan, Michelle (1997): Modes of Business Governance: European Management Styles and Corporate Cultures, in: West European Politics 20, S. 1-21.

Fama, Eugene (1980): Agency Problems and the Theory of the Firm, in: Journal of Political Economy 88, S. 288-307.

Faust, Michael (2000): Warum boomt die Managementberatung? - Und warum nicht zu allen Zeiten und überall?, in: SOFI-Mitteilungen 28/2000, S. 59-85.

Galbraith, John Kenneth (1967): Die moderne Industriegesellschaft, München/Zürich: Droemersche Verlagsanstalt.

Hartmann, Heinz (1968): Der deutsche Unternehmer: Autorität und Organisation, Frankfurt: Europäische Verlagsanstalt.

Hartmann, Michael (1995): Deutsche Topmanager: Klassenspezifischer Habitus als Karrierebasis, in: Soziale Welt 46, S. 440-468. 
Hartmann, Michael (1997): Die Rekrutierung von Topmanagern in Europa. Nationale Bildungssysteme und die Reproduktion der Eliten in Deutschland, Frankreich und Großbritannien, in: Archives Europeennes de Sociologie 38, S. 3-37.

Hartmann, Michael (2004): Eliten in Deutschland. Rekrutierungswege und Karrierepfade, in: Aus Politik und Zeitgeschichte B 10/2004, S. 17-24.

Höpner, Martin/Krempel, Lothar (2003): The Politics of the German Company Network. MPIfG Working Paper 2003/4, Köln: Max-Planck-Institut für Gesellschaftsforschung.

Höpner, Martin (2003): Wer beherrscht die Unternehmen? Shareholder Value, Managerherrschaft und Mitbestimmung in Deutschland, Frankfurt: Campus.

Höpner, Martin (2004): Unternehmensmitbestimmung unter Beschuss. Die Mitbestimmungsdebatte im Licht der sozialwissenschaftlichen Forschung, in: Industrielle Beziehungen 11, S. 347-379.

Januszewski, Silke I./Köke, Jens F. /Winter, Joachim K. (1999): Product Market Competition, Corporate Governance and Firm Performance: An Empirical Analysis for Germany. ZEW Discussion Paper 9963, Mannheim: Zentrum für Europäische Wirtschaftsforschung.

Jensen, Michael C. (1993): The Modern Industrial Revolution, Exit, and the Failure of Internal Control Systems, in: The Journal of Finance 48, S. 831-880.

Jensen, Michael C./Meckling, William (1976): Theory of the Firm: Managerial Behavior, Agency Costs and Ownership Structure, in: Journal of Financial Economics 3, S. 305-360.

Kraft, Kornelius/Niederprüm, Antonia (1999): Determinants of Management Compensation with RiskAverse Agents and Dispersed Ownership of the Firm, in: Journal of Economic Behavior and Organization 40, S. 17-27.

Lane, Christel (1989): Management and Labour in Europe. The Industrial Enterprise in Germany, Britain and France, Aldershot: Gower.

Monopolkommission (1976): Mehr Wettbewerb ist möglich. Erstes Hauptgutachten der Monopolkommission, Baden-Baden: Nomos.

Monopolkommission (1998a): Marktöffnung umfassend verwirklichen. Zwölftes Hauptgutachten der Monopolkommission, Baden-Baden: Nomos.

Monopolkommission (1998b): Ordnungspolitische Leitlinien für ein funktionsfähiges Finanzsystem. Sondergutachten der Monopolkommission, Baden-Baden: Nomos.

Naßmacher, Karl-Heinz (1997): Banken als Thema öffentlicher Politik, in: Der Bürger im Staat 47/1, S. 3-10.

Norton, Gary/Schmid, Frank A. (1996): Universal Banking and the Performance of German Firms. NBER Working Paper 5453, Cambridge, MA: National Bureau of Economic Research.

Perlitz, Manfred/Seger, Frank (1994): The Role of Universal Banks in German Corporate Governance, in: Business \& The Contemporary World 4, S. 49-67.

Poensgen, Otto H. (1982): Der Weg in den Vorstand. Die Charakteristiken der Vorstandsmitglieder der Aktiengesellschaften des verarbeitenden Gewerbes, in: Die Betriebswirtschaft 42, S. 3-25.

Rappaport, Alfred (1999): Shareholder Value. Ein Handbuch für Manager und Investoren. Übersetzt von Wolfgang Klien. 2., vollständig überarbeitete und aktualisierte Auflage, Stuttgart: Schäffer-Poeschel.

Rubach, Michael J./Sebora, Terrence C. (1998): Comparative Corporate Governance, in: Journal of World Business 33, S. 167-184.

Schmid, Frank A. (1996): Banken, Aktionärsstruktur und Unternehmenssteuerung, in: Kredit und Kapital 29, S. 402-427 und S. 545-564.

Schmid, Frank A. (1997): Vorstandsbezüge, Aufsichtsratsvergütung und Aktionärsstruktur, in: Zeitschrift für Betriebswirtschaft 67, S. 67-83.

Shleifer, Andrei/Vishny, Robert W. (1996): A Survey of Corporate Governance. NBER Working Paper 5554, Cambridge, MA: National Bureau of Economic Research. 
Sorge, Arndt (1999): Mitbestimmung, Arbeitsorganisation und Technikanwendung. In: Wolfgang Streeck/Norbert Kluge (Hrsg.), Mitbestimmung in Deutschland. Tradition und Effizienz. Expertenberichte für die Kommission Mitbestimmung, Frankfurt: Campus, S. 17-134.

Steward, Rosemary/Barsoux, Jean-Louis/Kieser, Alfred/Ganter, Hans-Dieter/Walgenbach, Peter (1994): Managing in Britain and Germany, London: Anglo-German Foundation for the Study of Industrial Society.

Thomsen, Steen/Pedersen, Torben (2000): Ownership Structure and Economic Performance in the Largest European Companies, in: Strategic Management Journal 21, S. 689-705.

Walgenbach, Peter/Kieser, Alfred (1995): Mittlere Manager in Deutschland und Großbritannien. In: Georg Schreyögg/Jörg Sydow (Hrsg.), Managementforschung 5. Empirische Studien, Berlin/New York: de Gruyter, S. 259-309.

Williamson, Oliver E. (1964): The Economics of Discretionary Behavior: Managerial Objectives in a Theory of the Firm, Englewood Cliffs, N.J.: Prentice Hall.

Windolf, Paul (2002): Die Zukunft des rheinischen Kapitalismus. In: Jutta Allmendinger (Hrsg.), Organisationssoziologie. KZfSS-Sonderband 42, S. 414-442.

Windolf, Paul/Beyer, Jürgen (1995): Kooperativer Kapitalismus - Unternehmensverflechtungen im internationalen Vergleich, in: Kölner Zeitschrift für Soziologie und Sozialpsychologie 47, S. 1- 36.

Zapf, Wolfgang (1965): Die deutschen Manager. Sozialprofil und Karriereweg. In: ders. (Hrsg.), Beiträge zur Analyse der deutschen Oberschicht, München: Piper, S. 136-149.

Dr. Martin Höpner

Max-Planck-Institut für Gesellschaftsforschung

Paulstr. 3

50676 Köln

Tel. 0221/2767-188

hoepner@mpifg.de

\section{Anhang: Regressionsgleichungen}

Gleichung $1(\mathrm{n}=39)$ : Vergütung pro Vorstandsmitglied 1996 (in Mio. DM) $=0.965$ (t-Wert: 9.856, $\mathrm{p}=.000)+0.000024 *$ Wertschöpfung (t-Wert: $2.688, \mathrm{p}=.011)+0.0876 *$ Jahre Managerherrschaft $(\mathrm{t}-$ Wert: $2.311, \mathrm{p}=.027) . \mathrm{R}^{2}$ (angepasst) $=.220$.

Gleichung $2(\mathrm{n}=40)$ : Vergütung pro Vorstandsmitglied 1996 (in Mio. DM) $=1.187$ (t-Wert: 11.029, $\mathrm{p}=.000)+0.000024 *$ Wertschöpfung (t-Wert: 2.487, $\mathrm{p}=.018)-0.07571 *$ Jahre mit Bankenvertreter als Aufsichtsratsvorsitzendem (t-Wert: $-1.808, \mathrm{p}=.079)$. $\mathrm{R}^{2}$ (angepasst) $=.166$.

Gleichung 3 ( $\mathrm{n}=39$ ): Steigerungsrate der Vorstandsvergütung zwischen 1996 und 1999 (in Prozent) = 157.185 (t-Wert: 4.526, $\mathrm{p}=.000)+0.0076 *$ Wertschöpfung (t-Wert: 3.817, $\mathrm{p}=.001)-10.711 *$ Anteil der externen Gewerkschaftsvertreter im Aufsichtsrat (in Prozent) (t-Wert: -3.927, p=.000). $\mathrm{R}^{2}$ (angepasst $)=.336$.

Gleichung 4 (n=39): Steigerungsrate der Vorstandsvergütung zwischen 1996 und 1999 (in Prozent) = $11.62(\mathrm{t}$-Wert: $.542, \mathrm{p}=.591)+0.00458 *$ Wertschöpfung (t-Wert: $2.276, \mathrm{p}=.029)+21.438 *$ Jahre mit Bankenvertreter als Aufsichtsratsvorsitzendem (t-Wert: 2.574, $\mathrm{p}=.014) . \mathrm{R}^{2}$ (angepasst) $=.199$.

Gleichung 5 ( $\mathrm{n}=39)$ : Steigerungsrate der Vorstandsvergütung zwischen 1996 und 1999 (in Prozent) = 127.467 (t-Wert: 3.796, p=.001) + 0.007664*Wertschöpfung (t-Wert: 4.159, p=.000) + 19.469*Jahre mit Bankenvertreter als Aufsichtsratsvorsitzendem (t-Wert: 2.793, $\mathrm{p}=.008)-10.226 *$ Anteil der externen Gewerkschaftsvertreter im Aufsichtsrat (in Prozent) ( $\mathrm{t}$-Wert: $-4.078, \mathrm{p}=.000) . \mathrm{R}^{2}$ (angepasst)=.442.

Gleichung $6(\mathrm{n}=39)$ : Vergütung pro Vorstandsmitglied 1999 (in Mio. DM) $=1.187$ (t-Wert: 11.029, $\mathrm{p}=.000)+0.0000253 *$ Wertschöpfung (t-Wert: 2.487, p=.018) $-0.07571 *$ Jahre mit Bankenvertreter als Aufsichtsratsvorsitzendem (t-Wert: $-1.808, \mathrm{p}=.079)$. $\mathrm{R}^{2}$ (angepasst) $=.166$. 